\title{
A Review on prolonging Life time of sensor network using Duty cycle and Network coding
}

\author{
Priyanka Trivedi1), Parul Kansal2)
}

\begin{abstract}
The key challenge while designing any wireless sensor network is to elevate the life time of network. To maximize network lifetime many factors are taken into consideration, such as reduction in power usage, selection of optimized route thus making it economically efficient accompanied with use of improved version of communication models \& advanced sets of instruction i.e. protocols. To avoid abbreviation of power in nodes, residual power is forwarded to every node. Formulation of congestion zone called bottle neck is controlled by cope algorithm comprising a combination of both duty cycle and network coding thus reduces loss of data. As a result network lifetime is increased by reducing node count and path count near the sink node. This results in abundant data transmission near the sink with same number of transmission, thus increasing efficiency of system with increased lifetime. The other two prime factor taken into consideration are the amount of packet delivered as well as its latency.
\end{abstract}

Keywords: Wireless sensor networks (WSNs), Bottle neck zone(BNZ), cope algorithm, duty cycle, network coding, packet latency, packet delivery ratio(PDR).

\section{Introduction}

In WSN, numbers of nodes usually termed as motes are placed such that it covers a wide range of area and are fused to a common point known as sink node meant to collect the entire data of a network. This network, having wireless sensors constitutes of self-configured node that can be assigned for vigilance purpose for localities which are generally out of reach, such as, dense forests, deserts, glaciers and deep seas etc [1][2]. Within sensor nodes, wireless transceiver meant for transmission purpose, is controlled by a micro controller to collect data in collection unit, with certain set of transducers is order to process and acquire data from marshalled region. The nodes can synchronize themselves to form a system, with different hopping rate thus attaining coherency in a network. In energy constrained wireless sensor network each sensor node has restricted battery power thus there is need of network lifetime enhancement.

Received(March 28, 2017), Review Result(1st: April 20, 2017, 2nd: May 28, 2017), Accepted(July 10, 2017)

${ }^{1}$ Dept. P.G student, Bipin Tripathi Kumaon Institute of Technology, Dwarahat, Almora, Uttarakhand priyankatrivedipt99@gmail.com

${ }^{2}$ Dept. Assistant Professor, ECE Deptt, Bipin Tripathi Kumaon Institute of Technology, Dwarahat, Almora, Uttarakhand kansal.parul@gmail.com 
In a sensor network, near end node consumes more energy than far end nodes. Limited number of resources, and their absence near sink node leads to formulation of bottle neck zone (BNZ), thus creating congestion \& reduces the performance of entire network. Thus bottle neck zone creation leads to depletion of energy of node so rapidly that a another problem named as energy hole problem gets arises in a network. Whenever a fault is introduced to the node inside bottle neck, it affect the system reliability with wastage of energy inside the mesh. The two popular technique are used to save energy is duty cycle which cut idle listening and other one is network coding which prevents overhearing of packets.

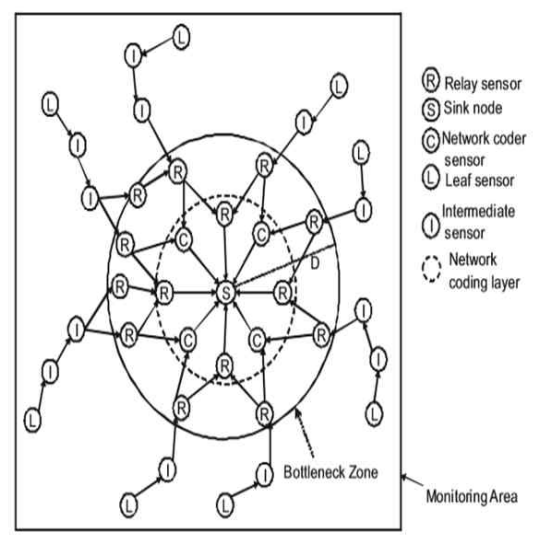

[Fig. 1] BNZ, role of sensors in typical WSN

To support long term application there is a need to build sustainable sensor network with increased network lifetime. Usually lifetime refers to the duration before the first node run out of energy. All node can't be practically active at time with limited power WSN, so toggling between hardworking and dormant (i.e. sleep) states saves energy. Duty cycle is calculated as the ratio between the time duration in which a sensor node is in active state to the total time of active/dormant states.

Two categories of duty cycle are there i.e. Random duty cycle [3] and Synchronized duty cycle. Sensor nodes switches to on and off state randomly in system having random duty cycle, whereas in synchronized duty cycle a coordination is maintained between the nodes via control message exchanges [4]. Duty cycle gives emphasis on connectivity and betterment of node placed over an area. Usually it is difficult to reduce traffic near the bottle neck zone, that's why upper bound of a lifetime is studied. For better utilization of bandwidth and data holding capability network ciphering is used which encode the packets by specified coding 
scheme before forwarding the coded packets to the next destination and increases reliability of network. This work can be accomplished in following ways.

Upper bound estimation [5] of network lifetime through bottle neck zone analysis (BNZ) can be done for Random duty cycle and non-duty cycle accompanied by network coding and without using network coding. Thus four possible result can be analyzed. Integration of duty cycle and network coding i.e. DU-NET coding utilize network resource more efficiently.

Simulation is done for proposed approach i.e. for network coding in term of packet efficiency including packet delivery ratio (PDR) and packet latency and also for increased network lifetime.

\subsection{Network Coding}

Network coding [7] is a way to encode facts packets obtained from its neighboring nodes, by intermediate node which are meant for encoding in mesh. To reduce the transmissions of different packets and efficient utilization of bandwidth random linear coding is used. Node store the packet which it receives and whenever there is a transmission opportunity, they send coded packet from linear combination. Poisson's [8] case is taken into consideration while transmitting packet streams. COPE technique is used as a base for the encoding and decoding process. Here XOR coding is used [9], thus reduces multiple transmission and avoid overhearing of packets when broadcasted in a network. The encoding of stream followed by decoding in receiving end [10][11] is done through linear network coding and is described as under.

Encipher operation: After coding the packet, for the purpose of transmission, node selects a sequence of coefficients $=(\mathrm{b} 1, \mathrm{~b} 2, . . ., \mathrm{bn})$, represents encoding vector, from $\mathrm{KL}\left(2^{\mathrm{s}}\right)$.

A set of packets $\mathrm{K}$ having $\mathrm{n}$ elements i.e. $\mathrm{Ki}(\mathrm{i}=1,2,3,4, \ldots, \mathrm{n})$ collected at a node are linearly

encoded into a one unique packet. The resultant encoded packet is denoted by

$$
\mathrm{Y}=\sum \mathrm{biKi}, \text { bi } € \mathrm{KL}\left(2^{\mathrm{s}}\right)
$$

Then the packets obtained after encoding with their $\mathrm{n}$ coefficients are forwarded further. The encoding vector contributes at the destination end in order to regain the encoded facts and figures i.e. for decoding packets to acquire the actual transmitted data.

Decoding operation: For reacquiring the true copy of packets one needs to solve certain set 
of linear equations at the receiving end. Let, a set $(\mathrm{b} 1, \mathrm{Y} 1) \cdots \cdots\left(\mathrm{b}^{\mathrm{m}}, \mathrm{Y}^{\mathrm{m}}\right)$ has been obtained by a node. The notation $Y_{j}$ is information symbol and $b j$ is the coding vector for the $j$ th obtained package. Decoding of packets is done at node by solving linear equation(2) having $\mathrm{n}$ unknowns and $m$ number of equation.

$$
Y_{j}=\sum \text { bij } K i, j=1, \cdots, m
$$

It is mandatory to receive $\mathrm{n}$ linearly independent coded packets for actual decoding of transmitted packets. The only unidentified, $\mathrm{Ki}$, comprises true packet that are transmitted in the network. After obtaining $\mathrm{n}$ linearly independent packets, $\mathrm{n}$ original packets can be re-acquired by solving certain set of linear equation. A type of linear network coding called XOR coding technique is used for this purpose. Elements in $\mathrm{KL}(2)=\{0,1\}$ are the coded packet that are transmitted in the network and bitwise XOR in $\mathrm{KL}(2)$ is utilized for processing.

\section{Related Works}

Duty cycle contributes to reduction in consumption of energy and power in a dense WSN accompanied with, network coding that results in improvement of throughput, bandwidth and power effectiveness. Examination of upper bound of network is done taking energy constrained nodes into consideration that collect data for a specified region .For cluster based network, its lifetime is estimated using a process called converge cast. Broadcasting scheme with reliability [6] is proposed and efforts are made to improvise the efficiency by reducing redundancy in broadcasting. When number of sensor node increase there is need to achieve over all synchronization and strict work sleep schedule. Studied for low duty cycled WSN is done in terms of coverage and connectivity [12]. For single unicast and single multicast connection and data transmission in a network, a coding scheme called random linear network coding have been proposed by Rout [13]. Network coding based broadcasting [14] focus or gives emphasis on reducing the number of transmission, by combining some of received message at forwarding node before transmission. Our prior work is to calculate the upper bounds value for lifetime time of entire network when random case of duty cycle is considered, second when network coding is taken into account and third when allegation of duty cycle \& network encoding is considered. A broad theoretical and analytical approach with simulation is applied to acquire the desired result. 


\section{Analysis of upper bound of life time of WSNs}

A network setup is examined with ' $\mathrm{N}$ ' sensor nodes placed uniformly in measured zone 'A'. BNZ of radius ' $\mathrm{D}$ ' over an area $\mathrm{A}$ is shown in Fig. 1. All node accounted with duty cycle, works as per name entitled illustrated in Fig. 1.For classification on functionality basis, we classifies nodes into two group one is relay sensor node, just used to route data to sink whereas other one i.e. mesh coded node is meant to be for coded operation. Usually small radius (D) are preferred as it provides an aid to route data generated outside the BNZ through the zone B.

Some common terms used in analysis are:

Node population(N); Area(A); radius of BNZ(D); Path loss exponent(n); $a_{11}$ (transmitter energy drain per bit); $a_{12}$ (sensor node energy drain per bit); $a_{2}$ (transmit op-amp energy drain per bit); Esleep (sleep state energy drain); Eb (total battery energy)

For efficient energy utilization, total energy drop in the bottle neck zone are categorized into three part: one for relay purpose i.e. to relay the data bit acquired from outside BNZ, second one is the sensing function in interior of $\mathrm{BNZ}$ and later one is to relay the facts and figures of the same.

Energy consumption by source node per second across a distance $d$ is

$$
E_{t x}=R_{d}\left(a_{11}+a_{2} d^{n}\right)
$$

Total energy consumption by a source node having duration $\mathrm{t}$ without acting as relay is

$$
\begin{aligned}
E s= & t[p(r s \text { es }+E t x)+(1-p) E \text { sleep }] \\
& \text { here } p \text { is duty cycle,rs is average sensing rate }
\end{aligned}
$$

Total energy consumption per second by intermediate node which actually working as a relay node

$$
E_{t x r}=R_{d}\left(a_{11}+a_{2} d^{n}+a_{12}\right)=R_{d}\left(a_{1}+a_{2} d^{n}\right)
$$

Total energy consumption by relay node 


$$
E_{R}=t\left[p\left(r_{s} e_{s}+E_{t x r}\right)+(1-p) E_{\text {Sleep }}\right]
$$

\section{a) Using duty cycle}

The upper bound of network life time when only duty cycle is considered is given as $\mathrm{Qx}(\mathrm{i})=\mathrm{p}(\mathrm{i})^{*}$ alpha1*(n/(n-1))*rs*(D*(A-B)*((m(j)+1)/2)+((2/3)

$\left.\left.{ }^{*} \mathrm{pi}^{*}(\mathrm{D})^{\wedge} 3\right)\right)+\mathrm{B}^{*} \mathrm{dm}{ }^{*}\left(\mathrm{p}(\mathrm{i})^{*} \mathrm{rs}^{*}(\mathrm{es}-\mathrm{alpha12})+(1-\mathrm{p}(\mathrm{i}))^{*}\right.$ Esleep);

$\mathrm{m}$ is number of active neighbor inside bottleneck zone.

b) considering duty cycle and network coding

Calculating upper bound with both parameter duty \& network coding, here $\mathrm{h}$ and $\mathrm{k}$ are the value of traffic flow through network coder node and relay node.

$$
\begin{aligned}
& \left.\mathrm{Qx}(\mathrm{i})=\mathrm{p}(\mathrm{i})^{*} \text { alpha1*(n/(n-1) }\right)^{*} \mathrm{rs}^{*}\left(\mathrm{D}^{*}(\mathrm{~A}-\mathrm{B})^{*}((\mathrm{~m}(\mathrm{j})+1) / 2)^{*}\left(\left(1+\mathrm{k}^{*}(\mathrm{~h}-1)\right) /\left(\mathrm{k}^{*} \mathrm{~h}\right)\right)+\left((2 / 3)^{*} \mathrm{pi}^{*}(\mathrm{D})^{\wedge} 3\right)\right)+\mathrm{B} \\
& { }^{*} \mathrm{dm}^{*}\left(\mathrm{p}(\mathrm{i})^{*} \mathrm{rs}^{*}(\mathrm{es}-\mathrm{alpha12})+(1-\mathrm{p}(\mathrm{i}))^{*} \text { Esleep }\right) \\
& \mathrm{t}(\mathrm{i})=\left(\mathrm{dm}^{*} \mathrm{~B}^{*} \mathrm{~Eb}\right) / \mathrm{Qx}(\mathrm{i})
\end{aligned}
$$

Using both network coding and duty cycle increases network life time to a large extent with less energy dissipation.

\section{Performance Analysis}

The selected parameter for energy utilization are based on MICA mode criteria [15].The duty cycle denoted by ' $\mathrm{p}$ ' of the WSN has been taken from one to ten percent. There is a tradeoff between duty cycle and network lifetime, if former one increases later one decreases. Increase in the duty cycle signifies that there is an increase in the number of nodes that are within active state in the mesh. Thus nodes are in working state with increased number of receptions and transmission in the network. Therefore, power usages tends to increase up to a large extent. It is analyzed that the lifetime time with DU-NET coding i.e. combination of duty cycle and network coding is more as compared to using duty cycle alone. There is a rapid rise of $2.5 \%$ to $9.5 \%$ of mesh lifetime by using above approach for $1 \%$ to $10 \%$ duty cycle respectively in the networked duty cycle WSN. The boost in the lifetime is due to the introduction of network coding, (here XOR a linear network coding is used.). Also there is an improvement in 
per node power consumption as compared to former case. The performance parameter such as packet density ratio (PDR) and packet latency are also analyzed.

\section{Conclusion}

In wireless sensor network (WSN), usually bottle neck zone is created around the sink. Thus, the bottle neck zone has a huge impact over the lifetime of a wireless sensor network. So network life time of upper bound is estimated using duty cycle, without using duty cycle and with combination of both network coding and duty cycle. It has been concluded that recommended approach provides reduction in power utilization in the BNZ and lead to reinforcement of network lifetime. Coding results reveal that coding for $1 \%$ to $10 \%$ duty cycle results an increase of $2.5 \%$ to $9.5 \%$ of network lifetime. Using Cope technique at different layer mainly forwarding layer, throughput of network gets increased without any pre- modification in routing process. The gain varies from few percent to multiple folds depending upon which transport protocol is used \& traffic pattern congestion level. It is concluded the per node energy utilization with active cycle is more than in comparison with DU-NET coding. This leads to obtain 50\% more data with equal energy utilization in the BNZ. The package latency and its consignment ratio at sink is investigated for the suggested approach, taking packet loss into account. Packet latency depends on node density and varies accordingly, thus high for low node density and there is drop in latency with increased number of nodes.

\section{REFERENCES}

[1] I. F. Akyildiz, W. Su. Y. Sankarsubramaniam and E. Cayirci, "Wireless sensor networks: a survey", Computer Network, Vol.38, No.4, pp.393-422, (2002).

[2] J. Yick, B. Mukherjee and D. Ghosal, "Wireless sensor network survey," Computer Network, Vol.52, No.12, pp.2292-2230, (2008).

[3] C. F. Hsin and M. Liu, "Randomly duty-cycled wireless sensor networks: dynamic of coverage," IEEE Trans. Wireless Commun., Vol.5, No.11, pp.3182-3192, (2006).

[4] X. Y. Wang, R. K. Dokania and A. Apsel, "PCO-based synchronization for cognitive duty-cycled impulse radio sensor networks," IEEE Sensors J., Vol.11, No.3, pp.555-563, (2011).

[5] Q. Wang and T. Zhang, "Bottleneck zone analysis in energyconstrained wireless sensor networks," IEEE Commun. Lett., Vol.13, No.6, pp.423-425, June (2009).

[6] D. Ganesan, R. Govindan, S. Shenker and D. Estrin, "Highly resilient energy efficient multipath routing in WSN" ACM SIGMOBILE mobile computing and commun. Rev., Vol.5, No.4, pp11-25, (2011). 
[7] R. Ahlswede, N. Cai, S. Y. R. Li and R. Yeung, "Network information flow", IEEE Trans. Inf. Theory, Vol.46, No.4., pp.1204-1216, July (2000).

[8] O. Lun, M. Mederard, R. Koettar and M. Effros, "On coding for reliable communication over packet network", Physical commun., Vol.1, pp.3-20, (2008).

[9] S. Katti, H. Rahul, W. Hu, D. Katabi, M. Medard, and J. Crowcroft, "XORs in the air:practical wireless network coding," IEEE Trans. Networking, Vol.16, pp.497-510, (2008).

[10] S. Lee and S. H. Lee, "Analysis of network lifetime in clusterbased sensor networks," IEEE Commun. Lett., Vol.14, No.10, pp. 900-902, (2010).

[11] Hamid Rafiei Karkvandi, Efraim Pecht, Orly Yadid-Pecht, "Effective lifetime-aware routing in wireless sensor networks," IEEE Sensors J., Vol.11, No.12, pp.3359-3367, (2011).

[12] D. Kim, C. F. Hsin and M. Liu, "Asymptotic connectivity of low dutycycled wireless sensor networks," in Proc. of IEEE MILCOM, pp.2441-2447, (2005)

[13] R. R. Rout and S. K. Ghosh, "Enhancement of Lifetime using duty cycle and network coding in wireless sensor networks", IEEE Trans. Wireless Commun., Vol.12, No.2, pp.656-667, (2013)

[14] S. Yang and J. Wu, "Efficient broadcasting using network coding and directional antennas in MANETs," IEEE Trans. Parallel Distrib. Syst., Vol.21, No.2, pp.148-161, (2010)

[15] Tripti Sharma, Brijesh Kumar, G. S. Tomar, "Performance Comparison of LEACH, SEP and DEEC Protocols in Wireless Sensor Network", UACEE International Journal of Advances in Computer Networks and its Security, Vol.2, Issue.1, pp.10-15, April (2012)

[16] J. Hill and D. Culler, "Mica: a wireless platform for deeply embedded networks," IEEE MICRO, Vol.22, No.6, pp.12-24, (2002).

[17] Jung-Yoon Kim, Tripti Sharma, Brijesh Kumar, G. S. Tomar, Karan Berry, Won-Hyung Lee, "Intercluster Ant Colony Optimization Algorithm for Wireless Sensor Network in Dense Environment", Hindawi International Journal of Distributed Sensor Network, Vol.2014, pp.1-8, (2014) 